\title{
Correlation between computed tomography findings and epidermal growth factor receptor and $K R A S$ gene mutations in patients with pulmonary adenocarcinoma
}

\author{
MASAYUKI SUGANO ${ }^{1}$, KIMIHIRO SHIMIZU $^{1}$, TETSUHIRO NAKANO ${ }^{1}$, SEIICHI KAKEGAWA ${ }^{1}$, \\ YOHEI MIYAMAE ${ }^{1}$, KYOICHI KAIRA ${ }^{2}$, TAKUYA ARAKI ${ }^{3}$, MITSUHIRO KAMIYOSHIHARA ${ }^{1}$, \\ OSAMU KAWASHIMA ${ }^{1}$ and IZUMI TAKEYOSHI ${ }^{1}$

\begin{abstract}
Departments of ${ }^{1}$ Thoracic and Visceral Organ Surgery, and ${ }^{2}$ Medicine and Molecular Science, Gunma University Graduate School of Medicine; ${ }^{3}$ Department of Pharmacy, Gunma

University Hospital, 3-39-15 Showa-machi, Maebashi, Gunma 371-8511, Japan
\end{abstract}

Received November 29, 2010; Accepted March 3, 2011

DOI: 10.3892/or.2011.1412

\begin{abstract}
We examined the correlation between computed tomography (CT) findings and the incidence of epidermal growth factor receptor $(E G F R)$ and KRAS mutations in lung adenocarcinoma. We analyzed the tumors of 136 patients with surgically resected primary lung adenocarcinoma. CT scans were evaluated for the presence of ground grass opacity (GGO), spiculation and the maximum diameter of the tumor was measured. SMart Amplification Process (ver. 2) was used to detect the presence of EGFR and KRAS mutations. EGFR and KRAS mutations were found in $56(41.1 \%)$ and $25(18.4 \%)$ of the 136 cases, respectively. Although no significant association was found between GGO and EGFR mutations ( $\mathrm{p}=0.07)$, the $E G F R$ mutation occurred more frequently in male patients with GGO than in those without GGO $(\mathrm{p}=0.04)$. The KRAS mutation occurred more frequently in patients whose tumor diameter was $\geq 31 \mathrm{~mm}$ than in those whose tumor diameter was $<30 \mathrm{~mm}(\mathrm{p}=0.003)$. Evaluation of CT findings may be helpful for determining the presence of EGFR and KRAS mutations, particularly when it is not possible to obtain a tumor specimen.
\end{abstract}

\section{Introduction}

Recently, several investigations have shown that mutations in the epidermal growth factor receptor $(E G F R)$ and the KRAS gene are significant prognostic factors for non-small cell lung cancer (NSCLC) (1-3). EGFR and KRAS mutations occur in

Correspondence to: Dr Kimihiro Shimizu, Department of Thoracic and Visceral Organ Surgery, Gunma University Graduate School of Medicine, 3-39-15 Showa-machi, Maebashi, Gunma 371-8511, Japan E-mail:kmshimizu@gmail.com

Key words: epidermal growth factor receptor, KRAS, pulmonary adenocarcinoma, computed tomography
8-10\% and $20-50 \%$ of NSCLC patients, respectively, whereas the EGFR mutation has been reported to occur in $27-56 \%$ of East Asian patients with NSCLC and the KRAS mutation occurs in $5-15 \%$ of those patients (1-5). Activating mutations in the tyrosine kinase domain of the EGFR gene are associated with sensitivity to EGFR tyrosine kinase inhibitors (TKIs), such as gefitinib and erlotinib (6). Gefitinib and erlotinib are synthetic small molecules that have been used to treat patients with unresectable or recurrent NSCLC, and the presence of EGFR mutations has been reported to be a good predictor of gefitinib and erlotinib effectiveness $(3,6)$. Moreover, NSCLC patients who are refractory to TKIs often harbor $K R A S$ mutations, suggesting that KRAS activation might confer TKI resistance by activating signaling pathways downstream of EGFR (2). Furthermore, these two mutations have been reported to be mutually exclusive (1). It is essential that physicians are able to readily identify these mutations to develop better therapeutic strategies.

EGFR and KRAS mutations have been detected in resected lung cancers using direct sequencing of DNA samples $(2,3)$. Recently, more sensitive methods have been used to detect EGFR mutations, such as the peptide nucleic acid-locked nucleic acid PCR clamp (PNA-LNA PCR clamp) and highresolution melting analysis (HRMA) (7-10). However, these methods are often not suitable for small biopsy specimens (11). We hypothesized that if the presence of the EGFR and $K R A S$ mutations were correlated with morphological features of the tumor that could be shown using diagnostic imaging modalities, such as computed tomography (CT) scans, mutation status could be predicted in patients who had unresectable lung cancer or relapse after surgery whose tissue could not be obtained for EGFR and KRAS mutation status analysis.

In the last decade, many investigators have reported that preoperative CT scan findings were related to pathological features and postoperative prognosis $(12,13)$. In the present study, we evaluated the internal tumor structure and ground glass opacity (GGO) using thin-section CT images.

Yano et al (14) reported a possible association between a high ratio of GGO components in small peripheral adeno- 
carcinoma and the presence of EGFR mutations, particularly among female patients. However, the correlation between CT findings and EGFR or KRAS mutation status has not been fully established. In particular, it is unclear whether a correlation exists between the CT findings in pulmonary adenocarcinoma and KRAS mutations. The aim of the present study was to evaluate the correlation between CT findings and EGFR and $K R A S$ mutation status in peripheral lung adenocarcinoma in a larger group of patients than previous studies.

\section{Patients and methods}

Patients. Surgically resected specimens of peripheral primary adenocarcinoma of the lung were obtained from 136 consecutive patients who underwent surgery at the Gunma University Hospital or Maebashi Red Cross Hospital between October 2002 and March 2008. After surgical removal, a portion of each sample was immediately frozen and stored at $-80^{\circ} \mathrm{C}$ until DNA extraction. Institutional approval was obtained, as was written informed from all patients.

CT findings. CT was performed using helical or multi-detector scanners. Conventional CT images were obtained serially with 7-10 mm section thickness and 7-10 mm section spacing. High-resolution CT (HRCT) images at the level of the tumor lesion were obtained serially with $1-2 \mathrm{~mm}$ section spacing. Images were reconstructed using a high-frequency algorithm and photographed with a window level of $-600 \mathrm{H}$ and a window width of $2000 \mathrm{H}$ as a 'lung window'. The following thin-section CT factors were evaluated by two independent clinicians: the maximum diameter of the tumor, the presence of spiculation and GGO. GGO was defined as a hazy increase in lung attenuation, without obscuring the underlying bronchial or vascular structures (15). Two observers who were unaware of the pathological staging, prognosis and gene mutation status reviewed each HRCT scan of the 136 patients and evaluated the GGO ratio of the tumor (GGO/tumor ratio; $\mathrm{G} / \mathrm{T}$ ratio), which was calculated using the maximum length of the GGO on the slices where the tumor was the largest. The G/T ratio was calculated as $\left(\mathrm{D}_{\mathrm{GGO}}-\mathrm{D}_{\mathrm{SOL}}\right) / \mathrm{D}_{\mathrm{GGO}}$, where $\mathrm{D}_{\mathrm{GGO}}$ was the largest area of the tumor, including the GGO, and $\mathrm{D}_{\mathrm{SOL}}$ was the largest solid area of the tumor excluding the GGO (Fig. 1). Additionally, the maximum diameter of the tumor and the presence of spiculation were evaluated. Discrepancies between the observers were resolved by discussion and recording of the measurements they agreed on.

EGFR and KRAS mutation analysis. Genomic DNA was extracted from an approximately 3-5 $\mathrm{mm}$ cube of tumor tissue using a DNA Mini Kit (Qiagen, Hilden, Germany), and serially diluted to $20 \mathrm{ng} / \mu \mathrm{l}$.

EGFR mutations in exons 19 and 21 were detected using a non-radioactive single-strand conformation polymorphism (Non-RI-SSCP) (7) and the SMart Amplification Process (ver. 2) (SmartAmp2) (16,17). The SMartAmp2 method is a unique genotyping technology that can detect a mutation in a single step in 30 min under isothermal conditions. It is based on strand-displacing DNA polymerase activity and can amplify and detect mutations directly from simple lung cancer sample preparations $(16,17)$.

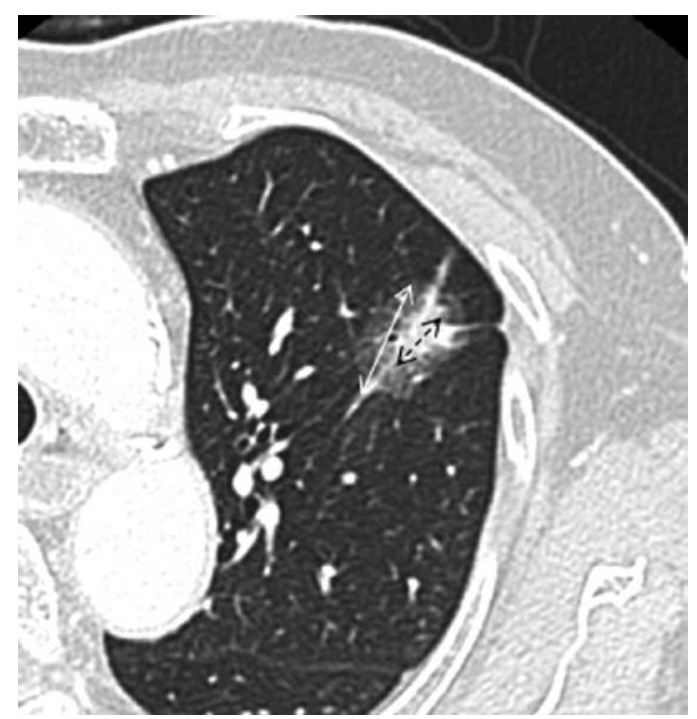

Figure 1. Computed tomography (CT) scan shows localized ground glass opacity (GGO) with increased attenuation at the center of the tumor in the left upper lobe of the lung. The maximum diameter of the GGO (continuous line) and the diameter of the solid part (dotted line) were determined using thin-section CT. The G/T ratio was calculated at $55 \%$.

The KRAS mutation was also detected using the SmartAmp2 assay using DNA extracted from tumor tissue (18). The EGFR and KRAS Mutation Detection Kit for SmartAmp2 assay was obtained from K.K. DNAFORM (Yokohama, Japan).

Statistical analysis. We were interested in any association between EGFR or KRAS mutations and various patient characteristics, including age, gender, smoking history, pathological stage of disease and CT findings. Unpaired t-tests were used to compare means and $\chi^{2}$ test and Fisher's exact test were used to compare proportions. A p-value of 0.05 was deemed to indicate statistical significance. Statistical analyses were carried out using the StatView software (ver. 5.0; SAS Institute, Cary, NC, USA).

\section{Results}

Patient characteristics associated with the EGFR and KRAS mutations. Table I shows the patient characteristics and EGFR mutation status. EGFR mutations were found in 56 (41.1\%) of the 136 cases. The mean age of the patients did not differ between the EGFR mutation and wild-type groups. $E G F R$ mutations were significantly more frequent in women than in men $(\mathrm{p}=0.005)$, and the wild-type gene was found significantly more often than the EGFR mutation in patients who had a history of smoking $(\mathrm{p}=0.0001)$. We observed no significant association between pathological cancer staging and the presence of the EGFR mutation.

Table II shows the patient characteristics and KRAS mutation status. We found that 25 (18.4\%) of the 136 cases had a KRAS mutation. No significant association was observed between the presence of the KRAS mutation and mean age $(\mathrm{p}=0.7)$, gender $(\mathrm{p}=0.25)$, smoking status $(\mathrm{p}=0.19)$ or pathological cancer staging $(\mathrm{p}=0.21)$. The KRAS mutation was detected only in patients who had the wild-type EGFR; thus, 
Table I. Patient characteristics and EGFR gene status.

\begin{tabular}{lccc}
\hline & $\begin{array}{c}\text { Mut }(\mathrm{n}=56) \\
\text { no. }(\%)\end{array}$ & $\begin{array}{c}\text { WT }(\mathrm{n}=80) \\
\text { no. }(\%)\end{array}$ & p-value \\
\hline Mean age (range) & $65(40-79)$ & $67(36-84)$ & 0.08 \\
Gender & & & \\
Male & $17(28)$ & $43(72)$ & 0.005 \\
Female & $39(53)$ & $37(47)$ & \\
Smoking history & & & \\
Yes & $15(24)$ & $48(76)$ & 0.0001 \\
No & $41(56)$ & $32(44)$ & \\
Pathological stage & & & \\
I & $40(40)$ & $59(60)$ & 0.84 \\
II & $5(50)$ & $5(50)$ & \\
III & $11(41)$ & $16(59)$ & \\
\hline
\end{tabular}

$E G F R$, epidermal growth factor receptor; Mut, mutation; WT, wildtype.

Table II. Patient characteristics and KRAS gene status.

\begin{tabular}{lccc}
\hline & $\begin{array}{c}\text { Mut }(\mathrm{n}=25) \\
\text { no. }(\%)\end{array}$ & $\begin{array}{c}\text { WT (n=111) } \\
\text { no. }(\%)\end{array}$ & p-value \\
\hline $\begin{array}{l}\text { Mean age (range) } \\
\text { Gender }\end{array}$ & $67(36-83)$ & $66(42-84)$ & 0.7 \\
Male & $13(22)$ & $47(78)$ & 0.25 \\
Female & $12(16)$ & $64(84)$ & \\
Smoking history & & & \\
Yes & $11(18)$ & $49(82)$ & 0.19 \\
No & $14(18)$ & $62(82)$ & \\
Pathological stage & & & \\
I & $16(16)$ & $83(84)$ & 0.21 \\
II & $1(10)$ & $9(90)$ & \\
III & $8(30)$ & $19(70)$ & \\
\hline
\end{tabular}

Mut, mutation; WT, wild-type.

our findings support previous reports indicating that the EGFR and KRAS mutations may be mutually exclusive.

Distribution of patients with EGFR and KRAS mutations according to $C T$ findings. The presence of spiculation, GGO and the maximum tumor diameter was assessed and the correlation with the mutations was determined (Table III). The EGFR mutation was detected in 35 (36\%) of the 96 tumors with spiculation and in 21 (52\%) of the 40 tumors with no spiculation. Although the frequency of EGFR mutations was higher in tumors with no speculation, it was not statistically significant $(\mathrm{p}=0.06)$. The maximum tumor diameter was $\leq 30 \mathrm{~mm}$ in $103(76 \%)$ of the 136 patients. Of those, 46 (45\%) harbored the EGFR mutation, whereas the mutation was found in 10 (30\%) of the 33 patients who had a maximum tumor diameter $\geq 31 \mathrm{~mm}$; this difference was not statistically significant $(\mathrm{p}=0.1)$. GGO was found in 59 (43\%) of 136 tumors and the EGFR mutation was present in $29(49 \%)$ of those. The EGFR mutation was found in $27(35 \%)$ of the 77 tumors with no GGO. Although the EGFR mutation was observed more frequently in tumors with GGO than in those with no GGO, the difference was not statistically significant $(\mathrm{p}=0.07)$. Table IV shows the correlation between GGO and EGFR mutation status and gender. GGO was observed in $36(47 \%)$ of 76 female patients and in $23(38 \%)$ of 60 male patients. GGO occurred more frequently in female patients than in male patients, but the difference was not statistically significant $(\mathrm{p}=0.11)$. In the female patients, $19(52 \%)$ of the tumors with GGO and $20(50 \%)$ of tumors with no GGO had EGFR mutations; this difference was not statistically significant $(\mathrm{p}=0.49)$. In male patients, $E G F R$ mutations were detected in $10(43 \%)$ of 23 tumors that had GGO, whereas the mutation was detected in $7(19 \%)$ of 37 tumors with no GGO. This difference was statistically significant $(\mathrm{p}=0.04)$. Thus, male patients with GGO had significantly more EGFR mutations than did those with no GGO.

The associations between the KRAS genotype and CT findings are summarized in Table III. KRAS mutations were found in $9(15 \%)$ of 59 tumors with GGO and in $16(21 \%)$ of 77 tumors without GGO. KRAS mutations were found in $19(20 \%)$ of 96 tumors with spiculation and in $6(15 \%)$ of 40 tumors without spiculation. The KRAS genotype did not correlate with the presence of spiculation or GGO $(\mathrm{p}=0.34$ and 0.27 , respectively). In contrast, KRAS mutations were found in $12(36 \%)$ of 33 tumors $>31 \mathrm{~mm}$ in diameter and in $13(13 \%)$ of 103 tumors $<30 \mathrm{~mm}$ in diameter $(\mathrm{p}=0.003)$. Thus, patients with a tumor $\geq 31 \mathrm{~mm}$ in diameter had a KRAS mutation significantly more frequently than did patients with a tumor $\leq 30 \mathrm{~mm}$. The results of the analysis of GGO and $K R A S$ mutation status in males and females are shown in Table IV. KRAS mutations were detected in three (13\%) of 23 male and $6(17 \%)$ of 36 female patients with GGO positive tumors, whereas KRAS mutations were detected in $10(27 \%)$ male and $6(15 \%)$ female patients with GGO negative tumors. No significant correlation was found between the presence of KRAS mutations and the existence of GGO in males $(\mathrm{p}=0.17)$ or females $(\mathrm{p}=0.69)$.

EGFR and KRAS mutations according to G/T ratio. Table V shows the EGFR and KRAS mutation status stratified by the $\mathrm{G} / \mathrm{T}$ ratio. Patients were divided into two groups according to $\mathrm{G} / \mathrm{T}$ ratio: 24 (18\%) patients with a $\mathrm{G} / \mathrm{T}$ ratio $\geq 50 \%$ and 112 $(82 \%)$ patients with a $\mathrm{G} / \mathrm{T}$ ratio $<50 \%$. In the 24 patients with a $\mathrm{G} / \mathrm{T}$ ratio $\geq 50 \%, E G F R$ mutations were found in $12(50 \%)$ of the tumors, whereas EGFR mutations were found in $44(39 \%)$ of the 112 tumors with a $\mathrm{G} / \mathrm{T}$ ratio $<50 \%$. EGFR mutations tended to occur more frequently in patients with a $\mathrm{G} / \mathrm{T}$ ratio $\geq 50 \%$ than in those with a $\mathrm{G} / \mathrm{T}$ ratio $<50 \%$; however, the difference was not statistically significant $(\mathrm{p}=0.22) . K R A S$ mutations were observed in two $(8 \%)$ of the 24 patients with a $\mathrm{G} / \mathrm{T}$ ratio $\geq 50 \%$ and in $23(21 \%)$ of 112 patients with a G/T ratio of $<50 \%$. Although the difference was not statistically significant, KRAS mutations were more frequently observed in patients with a $\mathrm{G} / \mathrm{T}$ ratio of $<50 \%$ than in those with a $\mathrm{G} / \mathrm{T}$ ratio of $\geq 50 \%(\mathrm{p}=0.13)$. When we further divided the 
Table III. Distribution of patients with EGFR and KRAS mutation according to CT findings.

\begin{tabular}{|c|c|c|c|c|c|c|}
\hline \multirow[b]{2}{*}{ CT findings } & \multicolumn{2}{|c|}{$E G F R$ genotype } & \multirow[b]{2}{*}{$\mathrm{p}$-value } & \multicolumn{2}{|c|}{$K R A S$ genotype } & \multirow[b]{2}{*}{$\mathrm{p}$-value } \\
\hline & Mut no. (\%) & WT no. (\%) & & Mut no. (\%) & WT no. $(\%)$ & \\
\hline Spiculation (+) & $35(36)$ & $61(64)$ & 0.06 & $19(20)$ & $77(80)$ & 0.34 \\
\hline Spiculation (-) & $21(52)$ & $19(48)$ & & $6(15)$ & $34(85)$ & \\
\hline Max diameter $\leq 30 \mathrm{~mm}$ & $46(45)$ & $57(55)$ & 0.1 & $13(13)$ & $90(87)$ & 0.003 \\
\hline Max diameter $\geq 31 \mathrm{~mm}$ & $10(30)$ & $23(70)$ & & $12(36)$ & $21(64)$ & \\
\hline GGO (+) & $29(49)$ & $30(51)$ & 0.07 & $9(15)$ & $50(85)$ & 0.27 \\
\hline GGO (-) & $27(35)$ & $50(65)$ & & $16(21)$ & $61(79)$ & \\
\hline
\end{tabular}

GGO, ground glass opacity; Mut, mutation; WT, wild-type.

Table IV. Correlation between GGO status and the EGFR and KRAS mutation in each gender.

\begin{tabular}{|c|c|c|c|c|c|c|}
\hline \multirow[b]{2}{*}{$\mathrm{G} / \mathrm{T}$ ratio } & \multicolumn{2}{|c|}{$E G F R$ genotype } & \multirow[b]{2}{*}{$\mathrm{p}$-value } & \multicolumn{2}{|c|}{$K R A S$ genotype } & \multirow[b]{2}{*}{ p-value } \\
\hline & Mut no. (\%) & WT no. (\%) & & Mut no. (\%) & WT no. (\%) & \\
\hline \multicolumn{7}{|l|}{ Male } \\
\hline GGO (+) & $10(43)$ & $13(57)$ & 0.04 & $3(13)$ & $20(87)$ & 0.17 \\
\hline GGO (-) & 7 (19) & $30(81)$ & & $10(27)$ & $27(73)$ & \\
\hline \multicolumn{7}{|l|}{ Female } \\
\hline GGO (+) & $19(52)$ & $17(48)$ & 0.49 & $6(17)$ & $30(83)$ & 0.69 \\
\hline GGO (-) & $20(50)$ & $20(50)$ & & $6(15)$ & $34(85)$ & \\
\hline
\end{tabular}

GGO, ground glass opacity; Mut, mutation, WT, wild-type.

Table V. Distribution of patients with $E G F R$ and $K R A S$ mutation stratified by G/T ratio.

\begin{tabular}{|c|c|c|c|c|c|c|}
\hline \multirow[b]{2}{*}{$\mathrm{G} / \mathrm{T}$ ratio $(\%)$} & \multicolumn{2}{|c|}{$E G F R$ genotype } & \multirow[b]{2}{*}{$\mathrm{p}$-value } & \multicolumn{2}{|c|}{$K R A S$ genotype } & \multirow[b]{2}{*}{$\mathrm{p}$-value } \\
\hline & Mut no. (\%) & WT no. $(\%)$ & & Mut no. (\%) & WT no. (\%) & \\
\hline$\geq 50$ & $12(50)$ & $12(50)$ & 0.22 & $2(8)$ & $22(92)$ & 0.13 \\
\hline$<50$ & 44 (39) & $68(61)$ & & $23(21)$ & 89 (79) & \\
\hline
\end{tabular}

GGO, ground glass opacity; G/T ratio, GGO/tumor ratio; Mut, mutation; WT, wild-type.

patients into five groups according to the G/T ratios of: $0 \%$, $1-25 \%, 26-50 \%, 51-75 \%$ and $76-100 \%$, we found no significant correlation between EGFR or KRAS mutation status and G/T ratio (data not shown).

\section{Discussion}

Recent studies have reported that EGFR mutations are often observed in adenocarcinomas, particularly among female patients and in Asian populations (6). More importantly, the presence of the EGFR mutation appears to be related to the patient's response to molecularly targeted drugs against $E G F R$ tyrosine kinase, such as gefitinib and erlotinib (19). Thus, detection of the EGFR mutation in lung adenocarcinomas is important for determining treatment strategy. In the present study, the SmartAmp2 assay or Non-RI-SSCP was used to detect EGFR mutations in 53 (42.4\%) of 136 patients who had adenocarcinomas. We found a significant correlation between EGFR mutation status and gender $(\mathrm{p}=0.005)$. Moreover, non-smoking was significantly associated with the presence of EGFR mutations ( $\mathrm{p}=0.0001$ ). Our findings confirmed those of previous reports showing an association between $E G F R$ mutations in lung adenocarcinomas and gender and smoking status $(1,6)$.

$K R A S$ is an oncogene that plays an important role in lung cancer carcinogenesis (20). KRAS mutations are found in 
$20-50 \%$ of lung adenocarcinomas $(1,4,5)$; however, the mutation is less common in Asian populations where it has been estimated to be $5-15 \%$ in adenocarcinomas $(5,21,22)$. We found KRAS gene mutations at codon 12 in 25 of the 136 adenocarcinoma patients $(18.5 \%)$. This percentage is higher than that previously reported $(5,21,22)$ and the difference may be the result of using the SmartAmp2 method to detect KRAS mutations; it is more sensitive than PCR-based direct sequencing, enzyme-enriched sequencing or PNA-enriched sequencing, used in previous reports (23). Thus, our method of detecting KRAS mutations had a higher sensitivity than did studies using alternative methods (16). KRAS mutations have previously been identified in adenocarcinomas and shown to have a significant association with gender and smoking (1). The $K R A S$ mutation has been detected more frequently in smokers and in male patients; however, recent studies suggest that KRAS mutations are not rare in never smokers or in women $(24,25)$, a finding confirmed by our results. The high sensitivity of the SmartAmp2 assay in detecting KRAS mutation may explain why we did not find a significant association between KRAS mutations and gender $(\mathrm{p}=0.25)$ or smoking status $(\mathrm{p}=0.19)$.

Several recent investigations have reported a correlation between the presence of EGFR and KRAS mutations and pathological findings, such as bronchioloalveolar cell carcinoma (BAC) or BAC features (3,7). Moreover, BAC or BAC features are known to have a particular appearance on CT scans including GGO (26,27); thus, we expected that the appearance of GGO on CT scans would predict the presence of EGFR or $K R A S$ mutations. It is often difficult to obtain tissue samples from patients with unresectable advanced lung cancer or who have had a relapse after surgery; thus, it would be clinically useful to be able to predict EGFR and KRAS mutation status using CT findings alone.

A relationship between $E G F R$ or KRAS mutation status and $\mathrm{CT}$ findings has been previously reported. Yano et al (14) reported that a high ratio of GGO components may predict the presence of EGFR mutations. They analyzed 80 peripheral adenocarcinomas, including 38 cases with $E G F R$ mutations, and found that GGO was more frequently observed in the mutation group than in the wild-type group, although the difference was not statistically significant. An analysis of the correlation between the EGFR mutation, nodule diameter and GGO ratio revealed that the $E G F R$ mutation frequency was significantly higher in patients who had a GGO ratio $\geq 50 \%$ and a tumor diameter $\leq 3 \mathrm{~cm}$ than it was in patients who had a GGO ratio of $<50 \%$ and a tumor diameter of $\leq 3 \mathrm{~cm}$. Female patients who had a GGO ratio $\geq 50 \%$ and a tumor diameter $<3 \mathrm{~cm}(10$ of 12 patients; $83 \%$ ) were most likely to have a high frequency of EGFR mutations (14). Although Yano et al did not find a significant association between GGO and EGFR mutation in all cases, they were able to identify the population with the highest frequency of EGFR mutations. Thus, we conducted our study using a larger sample size, a new system for analyzing $E G F R$ mutations and different algorithms for CT findings.

Glynn et al (28) found no correlation between morphological features on a CT scan and the presence of EGFR or KRAS mutations. They studied 77 lesions from 64 patients with lung adenocarcinoma with bronchioloalveolar features. They found that 10 of 26 nodules with a GGO lesion had EGFR mutations and nine nodules had KRAS mutations. They concluded that the presence of GGO on the CT scan was not significantly associated with the presence of an EGFR (p=0.44) or KRAS mutation ( $\mathrm{p}=0.77)$. However, Glynn et al only analyzed the presence of GGO and did not take into account the proportion of GGO components in the tumors. Furthermore, their sample size (26 nodules with GGO) was relatively small. Their method of analyzing GGO and a small sample size may help explain why they did not find a statistically significant correlation between CT findings and mutation status.

Our study was conducted using a simpler evaluation of CT findings and a larger group of patients than those used by Yano et al (14) or Glynn et al (28). We initially evaluated the presence of GGO in each tumor. We observed that the tumors with GGO tended to have EGFR mutations more frequently than did those with no GGO, though the difference did not reach statistical significance $(\mathrm{p}=0.07)$. This result suggested a possible correlation between the presence of GGO in a tumor and EGFR mutation status. EGFR mutations were detected in more than half of the female patients. The frequency of $E G F R$ mutations was significantly higher in female patients than males; thus, we further examined the gender difference in the correlation between CT findings and EGFR mutation. In female patients, we found no difference in $E G F R$ mutations status regardless of GGO $(\mathrm{p}=0.49)$. However, male patients with GGO had significantly more EGFR mutations than did those without GGO $(\mathrm{p}=0.04)$. The gender difference in the association between EGFR mutation status and CT findings suggests that it may be possible to predict the presence of EGFR mutations from CT findings in male patients. A similar tendency has been observed in the correlation between EGFR mutation status and pathological findings $(26,27)$. Haneda et al (29) reported a correlation between EGFR mutation status and BAC in male Japanese patients who had adenocarcinomas, but found no such correlation in female patients. The frequency of EGFR mutations was higher in male adenocarcinoma patients with BAC than in those with no BAC (52 vs. $21 \%$; p=0.013). Our results also revealed a gender difference in the correlation between $E G F R$ status and the morphological structure of the tumors. EGFR mutations play a different role in the carcinogenesis of lung adenocarcinoma in male and female patients and the gender-related mechanism may influence the correlation between pathological findings and EGFR status (30,31). Several investigators have reported gender-related differences in the behavior of lung cancer as a result of hormonal effects on the tumor $(30,31)$. They suggested that the effect was mediated by cross-talk between EGFR and estrogen receptors in the lung adenocarcinoma (32). This may partially explain the genderrelated difference in the role of EGFR in the oncogenesis of lung adenocarcinoma.

We used the G/T ratio, which was calculated as the tumorlength ratio, in our analyses rather than the GGO ratio, calculated as the tumor area ratio, used by Yano et al (14). We calculated the tumor-length ratio from the maximum length of the solid portion and the GGO in the tumor (Fig. 1), which can be determined readily in the clinical setting. The tumor-length ratio has been reported to be approximately the same value as the tumor area ratio and tumor volume ratio (33). We observed a tendency for the EGFR mutation to occur in tumors with GGO that suggests a close association between $\mathrm{G} / \mathrm{T}$ ratio and the frequency of EGFR mutation. We divided patients into two 
groups according to $\mathrm{G} / \mathrm{T}$ ratio: $\mathrm{G} / \mathrm{T}$ ratio $\geq 50 \%$ and $\mathrm{G} / \mathrm{T}$ ratio $<50 \%$, similar to Yano et al. We found that EGFR mutations tended to occur more frequently the patients with a $\mathrm{G} / \mathrm{T}$ ratio $\geq 50 \%$ than in those with a $\mathrm{G} / \mathrm{T}$ ratio $<50 \%$, although the difference was not statistically significant $(p=0.22)$. When we further divided the patients into five groups according to $\mathrm{G} / \mathrm{T}$ ratio we did not find a significant correlation between EGFR mutation status and $\mathrm{G} / \mathrm{T}$ ratio. These results suggest that the EGFR mutation status is more strongly correlated with the presence of GGO than with the proportion of GGO components.

A correlation between CT findings and the KRAS mutation was not established by Glynn et al (28). We found that $K R A S$ mutations occurred significantly more frequently in patients who had a tumor $>31 \mathrm{~mm}$ in diameter than in patients who had a tumor $\leq 30 \mathrm{~mm}$. The KRAS mutation is thought to be an early event that plays a role in tumor initiation $(34,35)$. Woo et al (36) reported that the KRAS mutation and a tumor size $>30 \mathrm{~mm}$ were independent prognostic factors in stage I lung adenocarcinoma (KRAS mutation; HR 4.51, 95\% CI 1.68-12.06, $\mathrm{p}=0.003$, tumor size $>30 \mathrm{~mm}$; HR $4.55,95 \% \mathrm{CI}$ $1.61-12.82, \mathrm{p}=0.004$, respectively). At the same time, they did not find a distinct correlation between the KRAS mutation and tumor size. Marks et al (25) analyzed nearly 300 cases of patients who had genotyped lung adenocarcinomas and had not received TKI therapy. They reported that patients with the KRAS mutation presented with significantly later stage disease than did patients with the EGFR-mutation and when compared with both EGFR mutant and wild-type combined. Furthermore, Marks et al reported that patients with KRAS mutant tumors had a shorter overall survival than did patients with $E G F R$ mutant tumors and vs. EGFR mutant tumors and $E G F R / K R A S$ wild-type tumors together. These findings indicate that the KRAS mutation has a strong influence on the acceleration of tumor progression as well as on the carcinogenesis of lung adenocarcinoma. Our finding that the $K R A S$ mutation occurred more frequently in larger tumors may reflect the effect(s) of the KRAS mutation.

Patients with BAC had been shown to be particularly responsive to EGFR TKIs (37). In a retrospective review of 139 patients treated with gefitinib, higher response rate were noted in adenocarcinoma with BAC features or pure BAC compared with other adenocarcinomas (38 vs. 14\%, p<0.001) (38). Zakowski et al reported that tumors from TKI responders tended to be better-differentiated adenocarcinomas with BAC components (39). They revealed that $78 \%$ of all responders had BAC or BAC components. Considering that BAC or BAC features are known to have a particular appearance on $\mathrm{CT}$ scans including GGO $(26,27)$, the recognition of the presence of GGO might result in more successful treatment with TKIs.

In conclusion, we found few correlations between $\mathrm{CT}$ findings and the presence of EGFR or KRAS gene mutations. We found that the $E G F R$ mutation occurred more frequently in male patients with GGO than in patients with no GGO $(\mathrm{p}=0.04)$. Although the frequency of $E G F R$ mutations was lower in male patients than in female patients, it may be possible to predict the presence of EGFR mutation using $C T$ findings. Although the genetic analysis of the resected specimen provides the most accurate evaluation of EGFR and $K R A S$ mutation status, the use of morphological features on a CT scan may be used to predict EGFR and KRAS mutation status in patients whose tumor specimens cannot be obtained.

\section{References}

1. Tam IY, Chung LP, Suen WS, et al: Distinct epidermal growth factor receptor and KRAS mutation patterns in non-small cell lung cancer patients with different tobacco exposure and clinicopathologic features. Clin Cancer Res 12: 1647-1653, 2006.

2. Paez JG, Janne PA, Lee JC, et al: EGFR mutations in lung cancer: correlation with clinical response to gefitinib therapy. Science 304: 1497-1500, 2004.

3. Lynch TJ, Bell DW, Sordella R, et al: Activating mutations in the epidermal growth factor receptor underlying responsiveness of non-small cell lung cancer to gefitinib. N Engl J Med 350: 2129-2139, 2004.

4. Mounawar M, Mukeria A, Le Calvez F, et al: Patterns of EGFR, HER2, TP53, and KRAS mutations of p14arf expression in non-small cell lung cancers in relation to smoking history. Cancer Res 67: 5667-5672, 2007

5. Suzuki M, Shigematsu H, Iizasa T, et al: Exclusive mutation in epidermal growth factor receptor gene, HER-2, and KRAS, and synchronous methylation of non-small cell lung cancer. Cancer 106: 2200-2207, 2006

6. Pao W and Miller VA: Epidermal growth factor receptor mutations, small-molecule kinase inhibitors, and non-small cell lung cancer: current knowledge and future directions. J Clin Oncol 23: 2556-2568, 2005.

7. Marchetti A, Martella C, Felicioni L, et al: EGFR mutations in non-small cell lung cancer: analysis of a large series of cases and development of a rapid and sensitive method for diagnostic screening with potential implications on pharmacologic treatment. J Clin Oncol 23: 857-865, 2005.

8. Nagai Y, Miyazawa H, Huqun, et al: Genetic heterogeneity of the epidermal growth factor receptor in non-small cell lung cancer cell lines revealed by a rapid and sensitive detection system, the peptide nucleic acid-locked nucleic acid PCR clamp. Cancer Res 65: 7276-7282, 2005.

9. Asano H, Toyooka S, Tokumo M, et al: Detection of EGFR gene mutation in lung cancer by mutant-enriched polymerase chain reaction assay. Clin Cancer Res 12: 43-48, 2006.

10. Nomoto K, Tsuta K, Takano T, et al: Detection of EGFR mutations in archived cytologic specimens of non-small cell lung cancer using high-resolution melting analysis. Am J Clin Pathol 126: 608-615, 2006.

11. Horiike A, Kimura H, Nishio K, et al: Detection of epidermal growth factor receptor mutation in transbronchial needle aspirates of non-small cell lung cancer. Chest 131: 1628-1634, 2007.

12. Hashizume T, Yamada K, Okamoto N, et al: Prognostic significance of thin-section CT scan findings in small-sized lung adenocarcinoma. Chest 133: 441-447, 2008.

13. Yoshida Y, Kokubu A, Suzuki K, et al: Molecular markers and changes of computed tomography appearance in lung adenocarcinoma with ground-glass opacity. Jpn J Clin Oncol 37: 907-912, 2007.

14. Yano M, Sasaki H, Kobayashi Y, et al: Epidermal growth factor receptor gene mutation and computed tomographic findings in peripheral pulmonary adenocarcinoma. J Thorac Oncol 1: 413-416, 2006.

15. Aoki T, Nakata $\mathrm{H}$, Watanabe $\mathrm{H}$, et al: Evolution of peripheral lung adenocarcinomas: CT findings correlated with histology and tumor doubling time. AJR Am J Roentgenol 174: 763-768, 2000.

16. Hoshi K, Takakura H, Mitani Y, et al: Rapid detection of epidermal growth factor receptor mutations in lung cancer by the SMart-Amplification Process. Clin Cancer Res 13: 4974-4983, 2007.

17. Mitani Y, Lezhava A, Kawai Y, et al: Rapid SNP diagnostics using asymmetric isothermal amplification and a new mismatchsuppression technology. Nat Methods 4: 257-262, 2007.

18. Tatsumi K, Mitani Y, Watanabe J, et al: Rapid screening assay for KRAS mutations by the modified smart amplification process. J Mol Diagn 10: 520-526, 2008.

19. Ciardiello F, De Vita F, Orditura M and Tortora G: The role of EGFR inhibitors in non-small cell lung cancer. Curr Opin Oncol 16: 130-135, 2004.

20. Slebos RJ, Kibbelaar RE, Dalesio O, et al: K-ras oncogene activation as a prognostic marker in adenocarcinoma of the lung. N Engl J Med 323: 561-565, 1990. 
21. Buttitta F, Barassi F, Fresu G, et al: Mutational analysis of the HER2 gene in lung tumors from Caucasian patients: mutations are mainly present in adenocarcinomas with bronchioloalveolar features. Int J Cancer 119: 2586-2591, 2006.

22. Finberg KE, Sequist LV, Joshi VA, et al: Mucinous differentiation correlates with absence of EGFR mutation and presence of KRAS mutation in lung adenocarcinomas with bronchioloalveolar features. J Mol Diagn 9: 320-326, 2007.

23. Araki T, Shimizu K, Nakamura K, et al: Usefulness of peptide nucleic acid (PNA)-clamp smart amplification process version 2 (SmartAmp2) for clinical diagnosis of KRAS codon 12 mutations in lung adenocarcinoma: comparison of PNA-clamp SmartAmp2 and PCR-related methods. J Mol Diagn 12: 118-124, 2010.

24. Riely GJ, Kris MG, Rosenbaum D, et al: Frequency and distinctive spectrum of KRAS mutations in never smokers with lung adenocarcinoma. Clin Cancer Res 14: 5731-5734, 2008.

25. Marks JL, Broderick S, Zhou Q, et al: Prognostic and therapeutic implications of EGFR and KRAS mutations in resected lung adenocarcinoma. J Thorac Oncol 3: 111-116, 2008.

26. Suzuki K, Asamura H, Kusumoto M, Kondo $H$ and Tsuchiya $R$ 'Early' peripheral lung cancer: prognostic significance of ground glass opacity on thin-section computed tomographic scan. Ann Thorac Surg 74: 1635-1639, 2002.

27. Asamura H, Suzuki K, Watanabe S, Matsuno Y, Maeshima A and Tsuchiya R: A clinicopathological study of resected subcentimeter lung cancers: a favorable prognosis for ground glass opacity lesions. Ann Thorac Surg 76: 1016-1022, 2003.

28. Glynn C, Zakowski MF and Ginsberg MS: Are there imaging characteristics associated with epidermal growth factor receptor and KRAS mutations in patients with adenocarcinoma of the lung with bronchioloalveolar features? J Thorac Oncol 5: 344-348, 2010

29. Haneda H, Sasaki H, Lindeman N, et al: A correlation between EGFR gene mutation status and bronchioloalveolar carcinoma features in Japanese patients with adenocarcinoma. Jpn J Clin Oncol 36: 69-75, 2006.
30. Nose N, Sugio K, Oyama T, et al: Association between estrogen receptor-beta expression and epidermal growth factor receptor mutation in the postoperative prognosis of adenocarcinoma of the lung. J Clin Oncol 27: 411-417, 2009.

31. Harichand-Herdt S and Ramalingam SS: Gender-associated differences in lung cancer: clinical characteristics and treatment outcomes in women. Semin Oncol 36: 572-580, 2009.

32. Marquez-Garban DC, Chen HW, Fishbein MC, Goodglick L and Pietras RJ: Estrogen receptor signaling pathways in human non-small cell lung cancer. Steroids 72: 135-143, 2007.

33. Aoki T, Tomoda Y, Watanabe H, et al: Peripheral lung adenocarcinoma: correlation of thin-section CT findings with histologic prognostic factors and survival. Radiology 220: 803-809, 2001.

34. Nakanishi H, Matsumoto S, Iwakawa R, et al: Whole genome comparison of allelic imbalance between non-invasive and invasive small-sized lung adenocarcinomas. Cancer Res 69: 1615-1623, 2009.

35. Soh J, Toyooka S, Ichihara S, et al: Sequential molecular changes during multistage pathogenesis of small peripheral adenocarcinomas of the lung. J Thorac Oncol 3: 340-347, 2008.

36. Woo T, Okudela K, Yazawa T, et al: Prognostic value of KRAS mutations and $\mathrm{Ki}-67$ expression in stage I lung adenocarcinomas. Lung Cancer 65: 355-362, 2009.

37. Fong T, Morgensztern D and Govindan R: EGFR inhibitors as first-line therapy in advanced non-small cell lung cancer. J Thorac Oncol 3: 303-310, 2008.

38. Miller VA, Kris MG, Shah N, et al: Bronchioloalveolar pathologic subtype and smoking history predict sensitivity to gefitinib in advanced non-small cell lung cancer. J Clin Oncol 22: 1103-1109, 2004

39. Zakowski MF, Hussain S, Pao W, et al: Morphologic features of adenocarcinoma of the lung predictive of response to the epidermal growth factor receptor kinase inhibitors erlotinib and gefitinib. Arch Pathol Lab Med 133: 470-477, 2009. 